\title{
Pneumococcal Vaccines: A Public Health Perspective
}

\author{
Mark Cohen ${ }^{1}$, Rafael Rodriguez ${ }^{2}$, Julio Ramirez ${ }^{3}$, Alfredo Guerreros ${ }^{4}$ and Sebastian Ospina-Henao ${ }^{5 *}$ \\ ${ }^{1}$ Pulmonary and Critical Care Department, Hospital Centro Medico, Guatemala \\ ${ }^{2}$ Faculty of Medicine, Universidad de Panama, Panama \\ ${ }^{3}$ Faculty of Medicine, Louisville University, United States of America \\ ${ }^{4}$ Faculty of Medicine, Universidad Nacional Mayor de San Marcos, Peru \\ ${ }^{5}$ Faculty of Medicine, Universidad de Ciencias Medicas (UCIMED), Costa Rica
}

Submission: February 18, 2022; Published: February 25, 2022

*Corresponding author: Dr. Sebastian Ospina-Henao, Instituto de Investigacion en Ciencias Medicas (IICIMED), Faculty of Medicine, Universidad de Ciencias Medicas (UCIMED), Costa Rica

\begin{abstract}
The Latin American Thoracic Association (ALAT, for its acronym in Spanish) is working to reinforce vaccination recommendations against pneumococcal disease, a disease that causes multiple consequences, from physical to social and economic, not only in children but also in adults. On November 24th, a forum of experts was held through Zoom platform. Issues such as: Disease burden, implementation recommendations, action steps, and scope of vaccination were discussed.

Keywords: Vaccines; Pneumococcal; Public Health; Pneumococcal Vaccines; COVID-19

Abbreviations: ALAT: Latin American Thoracic Association; GBD: Global Burden of Disease; CAP: Community-Acquired Pneumonia; EPI: Expanded Program on Immunization; WHO: World Health Organization; WHA: World Health Assembly; PPSV23: 23-Component Pneumococcal Polysaccharide Vaccine; ACIP: Advisory Committee on Immunization; CDC: Practices Center for Disease Control and Prevention; PCV13: 13-Component Pneumococcal Conjugate Vaccine; FDA: Food and Drug Administration
\end{abstract}

\section{Introduction}

Streptococcus pneumoniae (pneumococcus) is a Grampositive, capsulated coccus [1] known to be associated with high morbidity and mortality worldwide, mainly among children and in low-income countries, which have less access to bacterial diagnosis and disease treatment. This pathogen is a major etiologic cause of acute otitis media, pneumonia, meningitis and septicemia, as well as co-infections and superinfections in high-risk groups. Pneumococci colonize the nasopharynx and this carriage precedes disease, being the main source of spread among people, especially in children [2,3]. Colonization is usually asymptomatic, but they can spread to the lower respiratory tract and cause pneumonia or migrate to other body sites where they cause invasive disease such as bacteremia and meningitis [4]; this colonization is dynamic and varies from $30-75 \%$ in healthy children (especially those attending day care centers) [5], to 20$30 \%$ in healthy adults [6].

Among the pathogens that colonize the respiratory tract, pneumococci cause the most disease, especially when risk factors are present, making them one of the leading causes of lower respiratory tract infections worldwide [4]. Lower respiratory tract infections and pneumonia are the deadliest communicable diseases worldwide, ranking as the fourth leading cause of death, with more than 4 million deaths per year [7,8]; the 2017 Global Burden of Disease (GBD) study [9], estimated that lower respiratory tract infection caused by influenza accounted for 9,459,000 hospitalizations and 145,000 deaths among all age groups, with the highest mortality rate among adults older than 70 years; meanwhile, for 2016, infection by S. pneumoniae was the leading cause of morbidity and mortality from lower respiratory infections worldwide, contributing to $1,189,937$ deaths [10].

\section{Pneumococcal Disease}

One of the major risk factors for severe pneumococcal disease is preceding viral infections, especially Influenza virus serotype A. Secondary pneumococcal infections occurring during or after a viral infection are usually associated with unfavorable clinical outcomes, whereas a combined influenza and pneumococcal 
infection may be a co-infection or a secondary bacterial infection after influenza; in the case of coinfections, these are recognized as an underlying etiology of hospital-acquired pneumonia and community-acquired pneumonia (CAP), the latter being an acute infection that represents a significant burden of disease, especially in those population groups that are most susceptible to developing it: elderly and people with chronic diseases [4].

A prospective study conducted in three South American cities reported that incidence rates of CAP are higher in adults older than 65 years; of the participants included in the study, $82.4 \%$ had at least one underlying disease and $48 \%$ had two or more [11]. The increased risk of pneumococcal disease associated with established risk factors has persisted in the era of widespread vaccination of children with pneumococcal conjugate vaccines. Rates of pneumococcal disease increased substantially among persons with more than one risk condition, and rates of disease were especially high among people with $\geq 3$ risk conditions for younger adults, with a rate of all-cause pneumonia 15.6 times higher than that of healthy persons [12].

Multimorbidity is the coexistence of two or more chronic diseases; comorbidity, the conceptual predecessor of multimorbidity, was originally defined by Feinstein as "any additional distinct clinical entity that has existed or may occur during the course of a patient having the index disease under study" [13]. The prevalence of multimorbidity is over $60 \%$ worldwide and is likely to be over $80 \%$ among people over 85 years old. The main causes of chronic disease death can be attributed to four major underlying risk factors: smoking, unhealthy eating habits, alcohol consumption and sedentary lifestyle [14], which results in a direct impact on people's functional status, poor life quality and overall risk of death.

\section{S. Pneumoniae Vaccines}

In the last decades, immunization and its related advances have contributed to global development not only scientifically but also socially and economically; six vaccine-preventable diseases were the initial focus of the Expanded Program on Immunization (EPI) more than four decades ago, and at least six new vaccines have increased the breadth of protection provided by immunization. The World Health Organization (WHO) 2030 Agenda for Immunization envisions "a world in which everyone, everywhere and at every age, benefits fully from vaccines to improve health and well-being," for which it has been developed in a co-creation process with the full country engagement to ensure that the vision, strategic priorities, and goals are aligned with country requirements [15]

Factors supporting the progress achieved with immunization include improvements in health service delivery infrastructure; active surveillance of vaccine-preventable diseases promoted by national, regional and global laboratories; community involvement in vaccination programs and awareness of the vaccine's social value; data collection, management, analysis and use through electronic systems; vaccine research and development capacity; and evidence-based policy decision making; data collection, management, analysis and use through electronic systems; vaccine research and development capacity; as well as sciencebased policy decision-making [15]; additionally, immunization has contributed to Millennium Development Goal 4: Reduce child mortality, according to data from the Inter-agency Group for Child Mortality Estimation (UN-IGME), the under-five mortality rate per 1000 live births decreased from 75.83 in 2000 to 36.63 in 2020 [16].

The worldwide expansion of immunization programs started in 1974 with the establishment of the EPI by the World Health Assembly (WHA) [17], by 1983 a 23-component pneumococcal polysaccharide vaccine (PPSV23) was licensed in the United States, which was subsequently recommended by the Advisory Committee on Immunization Practices (ACIP) of the Center for Disease Control and Prevention (CDC), for all persons aged $\geq 65$ years, as well as for those aged $\geq 2$ years with chronic diseases associated with an increased risk of pneumococcal infection or complications of pneumococcal infection [3,12]. In 2011, the 13-component pneumococcal conjugate vaccine (PCV13) was licensed in the United States, and the ACIP recommended in 2012 that adults aged $\geq 19$ years with immunocompromising conditions, anatomic or functional asplenia, cerebrospinal fluid leaks, or cochlear implants receive PCV 13 followed by PPSV23 [18].

In September 2014, the ACIP reviewed its pneumococcal vaccination recommendation for adults aged 17 years and older to include the use of PCV13 in all adults aged $\geq 65$ years, this update boosted the use of PPSV23 with PCV13 in this population [19]; following this recommendation, a group of scientists conducted the first real-world data study, to evaluate the efficacy of PCV13 against hospital-type CAP in this population, using a negative test design, enrolled 2034 patients between cases and controls and concluded that the vaccine effectiveness of PCV13 against CAP in hospitalized population was 73\% [20]. The most recent approval was in June 2021 when the US Food and Drug Administration (FDA) approved the 20-element conjugate vaccine for the prevention of invasive disease and pneumonia caused by 20 serotypes of S. pneumoniae in adults aged $\geq 18$ years [21].

Another study [22] evaluated the efficacy of PCV13 in the prevention of first episodes of nonbacteremic and noninvasive community-acquired pneumonia and invasive pneumococcal disease vaccine strains, demonstrating that, among adults older than 65 years, PCV13 was effective in preventing the mentioned pathologies, maintaining such efficacy for at least four years, but not in preventing CAP due to any cause; being in accordance with other studies that have shown that, although purified capsular polysaccharides are effective against invasive pneumococcal disease in adults, their efficacy against non-invasive pneumonia is uncertain. 
In March 2017, WHO held a meeting to identify policies and activities to promote access to vaccination for older adults, specifically in low- and middle-income countries [23], the discussion at the meeting highlighted many reasons and benefits for vaccinating older adults and any age group after childhood; regarding these reasons, they raised three core pillars:

I. Improvement in population immunity (herd) and disease control, by updating the coverage of the complete childhood vaccination schedule when it is not $100 \%$, as well as the application of vaccines not used in adults when they were children (e.g., Measles, Rubella, Mumps, Hepatitis B, Varicella Zoster, Pneumococcal conjugate), and finally, in order to protect against pathogens with new strains or serotypes that may differ by age group (e.g., Influenza and Pneumococcus).

II. Protection of at-risk populations after childhood, this point is aimed at protection against pathogens associated with specific careers or employments, such as health care workers and farm workers with food products or in contact with animals. Also considered under this item is the prevention of hospitalized patient infections and the high morbidity and mortality rate of respiratory diseases in adults.

III. Protection throughout life, with emphasis on the protection of women during and after pregnancy to protect themselves and their babies, as well as vaccination as a primary measure to prevent the unnecessary use of antibiotics and avoiding the increase of antibiotic resistance; also proposed was the vaccination of travelers and vaccination to protect against emerging diseases, outbreaks and pandemics.

Regarding the barriers to adult vaccination in low- and middleincome countries, the experts discussed five central issues:

i. Evidence for action, with loss of burden disease economic data, recommendations in vaccination schedules and public policies issued on the subject.

ii. Individual factors and lack of information about vaccination to the population, leading to vaccine reticence.

iii. Vaccination process with very complex vaccination schedules in adults, with unplanned logistics where people must schedule several visits to obtain the prescription, supplies and then the immunization. Additional prioritization of children's immunization schedules.

iv. Vaccination system with limitations in infrastructure and human resources, lack of technical equipment to support vaccination campaigns, as well as lack of resources for the purchase and distribution of vaccines in adults, urban centralization of vaccination posts and lack of training for health personnel.

v. Community perception of adult health, since, in certain circumstances, society places the elderly's health in an inferior position compared to children's health.

\section{Pneumococcus and COVID-19}

The COVID-19 pandemic has highlighted the vulnerability of immunization programs, an essential health service, opening the door for resurgences of vaccine-preventable diseases and further complicating the pandemic situation. In the first quarter of 2020 , severe declines in immunization coverage, ranging from $10 \%$ to $50 \%$ compared to 2019 , occurred due to the implementation of severe social and physical distancing policies in many countries around the world [15]. The literature about the safety or efficacy of pneumococcal vaccine in the context of the COVID-19 pandemic is limited; in a cohort study of 11,672 people, 818 of whom had COVID-19, scientists found that people who had been given a pneumococcal vaccine in the same year as the study, had a lower risk of contracting COVID-19 [24], concluding that pneumococcal vaccination reduces the incidence of hospitalizations for pneumonia in adults over 65 years old, which is important because elderly people with COVID-19 infection have the highest risk of mortality [25].

In a retrospective study of 257 patients with laboratoryconfirmed COVID-19, bacterial coinfections were observed to be dominant in all patients with COVID-19, with S. pneumoniae being the most common pathogen, suggesting that pneumococcal conjugate and polysaccharide vaccines offer an effective approach to prevent the most common coinfection during the COVID-19 pandemic [26]. It is likely that pneumococcal vaccination can mitigate the impact of COVID-19 on healthcare systems by reducing morbidity and mortality due to non-COVID-19 respiratory infections, especially in those with significant risk factors such as advanced age and multimorbidity. It has been estimated that there are $10 \%$ of preventable COVID-19 deaths due to pneumococcal superinfection; these deaths could potentially have been prevented with vaccination [24].

\section{Conclusion}

The "real world" vision offered by vaccination efficacy data is essential when planning public health initiatives, and in the pneumococcal vaccines case it is essential to analyze the adult population, removing the paradigm that vaccines are only for children, opening it to the fact that non-vaccination in adults represents a significant disease burden and therefore requires their implementation, which combined with the vaccination of minors, becomes a family prevention strategy. Additionally, it should be taken into account that respiratory disease vaccination, particularly against pneumococcus, has a positive impact not only on the population' s health, but also on the resources assigned to it.

The COVID-19 pandemic, in its almost 2 years, has demonstrated that vaccines are powerful weapons for the infectious disease control, and that pneumococcal vaccination is fundamental for the control of the pandemic, as it decreases the 
burden of pneumonia disease, releasing resources and increasing the capacity for COVID care, in addition to protecting against complications due to pneumococcal co-infection. The pandemic also presents an opportunity for co-administration of vaccines, due to the fact that the risk groups for COVID-19, pneumococcal disease and influenza are similar. Finally, educating the population and raising awareness about the importance of vaccines will be reflected in fewer missed opportunities for vaccination and higher coverage in the target groups.

\section{Acknowledgements}

We would like to express our great appreciation to Pfizer for their valuable support for the publication of this manuscript, clarifying the total independence of the scientific content.

\section{References}

1. Prado JV (2001) Conceptos microbiologicos de Streptococcus pneumoniae: basic microbiological aspects. Rev Chil Infect P: 18.

2. Simell B, Auranen K, Kayhty H, Goldblatt D, Dagan R, et al. (2012) The fundamental link between pneumococcal carriage and disease. Exp Rev of Vac 11(7): 841-855.

3. Centers for Disease Control and Prevention (CDC) (1984) Update: pneumococcal polysaccharide vaccine usage-United States. MMWR 33(20): 273-276.

4. Sender V, Hentrich K, Henriques-Normark B (2021) Virus-Induced Changes of the Respiratory Tract Environment Promote Secondary Infections with Streptococcus pneumoniae. Front Cell Infect Microbiol 11: 643326

5. Hjalmarsdottir MA, Gumundsdottir PF, Erlendsdottir H, Kristinsson KG, Haraldsson G (2016) Cocolonization of Pneumococcal Serotypes in Healthy Children Attending Day Care Centers: Molecular Versus Conventional Methods. Pediatr Infect Dis J 35(5): 477-480.

6. Lindstrand A, Galanis I, Darenberg J, Eva M, Pontus N, et al. (2016) Unaltered pneumococcal carriage prevalence due to expansion of non-vaccine types of low invasive potential 8 years after vaccine introduction in Stockholm, Sweden. Vaccine 34(38): 4565-4571.

7. (2020) The Global Impact of Respiratory Disease. World Health Organization.

8. (2020) The top 10 causes of death. World Health Organization.

9. GBD 2017 Influenza Collaborators (2019) Mortality, morbidity, and hospitalisations due to influenza lower respiratory tract infections 2017: an analysis for the Global Burden of Disease Study 2017. Lancet Respir Med 7(1): 69-89.

10. GBD 2016 Lower Respiratory Infections Collaborators (2018) Estimates of the global, regional, and national morbidity, mortality, and aetiologies of lower respiratory infections in 195 countries 1990-2016: a systematic analysis for the Global Burden of Disease Study 2016. Lancet Infect Dis 18(11): 1191-1210.

11. Lopardo GD, Fridman D, Raimondo E, Henry A, Ana L, et al. (2018) Incidence rate of community- acquired pneumonia in adults: a population-based prospective active surveillance study in three cities in South America. BMJ 8(4): e019439.
12. Shea KM, Edelsberg J, Weycker D, Farkouh RA, Strutton DR, et al. (2014) Rates of Pneumococcal Disease in Adults with Chronic Medical Conditions. Open Forum Inf Dis 1(1): ofu024.

13. Feinstein AR (1970) The pre-therapeutic classification of co-morbidity in chronic disease. J Chronic Dis 23(7): 455-468.

14. Salive ME (2013) Multimorbidity in Older Adults. Epidem Reviews 35 75-83.

15. Lindstrand A, Cherian T, Chang-Blanc D, Feikin D, L. O'Brien K (2021) The World of Immunization: Achievements, Challenges, and Strategic Vision for the Next Decade. The Journal of Infectious Diseases 224(4): S452-S467.

16. (2020) Under-five mortality rate total. UN Interagency Group for Child Mortality Estimation IGME.

17. Okwo-Bele JM, Cherian T (2011) The expanded programme on immunization: A lasting legacy of smallpox eradication. Vaccine. 29 (4): D74-D79.

18. Centers for Disease Control and Prevention (CDC) (2012) Use of 13-valent pneumococcal conjugate vaccine and 23-valent pneumococcal polysac- charide vaccine for adults with immunocompromising conditions: recommendations of the Advisory Committee on Immunization Practices (ACIP). MMWR 61(40): 816-819.

19. Tomczyk S, Bennett NM, Stoecker C, Ryan G, Matthew RM, et al. (2014) Use of 13-valent pneumococcal conjugate vaccine and 23-valent pneumococcal polysaccharide vaccine among adults aged $\geq 65$ years: recommendations of the Advisory Committee on Immunization Practices (ACIP). MMWR 63(37): 822-825.

20. McLaughlin JM, Jiang Q Isturiz RE, Heather LS, David LS, et al. (2018) Effectiveness of 13-Valent Pneumococcal Conjugate Vaccine Against Hospitalization for Community-Acquired Pneumonia in Older US Adults: A Test-Negative Design. Clin Infect Dis 67(10): 1498-1506.

21. (2021) U.S. FDA Approves PREVNAR 20 $0^{\mathrm{TM}}$, Pfizer's Pneumococcal 20-valent Conjugate Vaccine for Adults Ages 18 Years or Older. Pfizer.

22. Bonten MJM, Huijts SM, Bolkenbaas M, Chris W, Scott P, et al. (2015) Polysaccharide Conjugate Vaccine against Pneumococcal Pneumonia in Adults. N Engl J Med 372: 1114-1125.

23. Aguado T, Barratt J, Beard JR, Bonnie BB, Wilbur HC, et al. (2018) Report on WHO meeting on immunization in older adults: Geneva, Switzerland, 22-23 March 2017. Vaccine 36(7): 921-931.

24. Lara J, Xinge J, Alex M, Serpil E, Brian PR, et al. (2020) Individualizing risk prediction for positive COVID-19 testing: results from $11,672 \mathrm{pa}-$ tients. Chest 158(4): 1364-1375.

25. Sultana J, Mazzaglia G, Luxi N, Antonino C, Annalisa C, et al. (2020) Potential effects of vaccinations on the prevention of COVID-19: rationale, clinical evidence, risks and public health considerations. Exp Rev Vac 19(10): 919-936.

26. Mendelson M (2020) Could enhanced influenza and pneumococcal vaccination programs help limit the potential damage from SARSCoV-2 to fragile health systems of southern hemisphere countries this winter?, Int J Infect Dis 94: 32-33. 
Your next submission with Juniper Publishers will reach you the below assets

- Quality Editorial service

- Swift Peer Review

- Reprints availability

- E-prints Service

- Manuscript Podcast for convenient understanding

- Global attainment for your research

- Manuscript accessibility in different formats ( Pdf, E-pub, Full Text, Audio)

- Unceasing customer service

Track the below URL for one-step submission https://juniperpublishers.com/online-submission.php 